\title{
Satisfaction and Experience of Palliative Patients with 24/7 Hotline Service During the COVID-19 Pandemic in Saudi Arabia
}

\author{
Sami Ayed Alshammary ${ }^{1 *}$, Yahya Assiri ${ }^{2}$, Reema AlRasheed ${ }^{3}$, Yacoub Abuzied ${ }^{4}$, Igbal Abelati Mahgoub ${ }^{5}$ and Khalid Alqumizi ${ }^{6}$ \\ ${ }^{1}$ Palliative Care Unit, Comprehensive Cancer Centre, King Fahad Medical City, Riyadh and Center for Postgraduate Studies in Family Medicine, \\ Ministry of Health, Riyadh, Saudi Arabia. \\ ${ }^{2}$ Department of North Business Unit, Health Holding Company, Riyadh, Saudi Arabia. \\ ${ }^{3}$ Department of Family and Community Medicine, PGY-1 Resident Physician, University of Toronto, Toronto, ON, Canada. \\ ${ }^{4}$ Department of Nursing, Rehabilitation Hospital, King Fahad Medical City, Riyadh, Saudi Arabia. \\ ${ }^{5}$ National Home for Health Services, Riyadh, Saudi Arabia. \\ ${ }^{6}$ Department of North Business Unit, Health Holding Company, Riyadh, Saudi Arabia.
}

* Corresponding Author: Sami Ayed Alshammary, Palliative Care Unit, Comprehensive Cancer Centre, King Fahad Medical City, Riyadh and Center for Postgraduate Studies in Family Medicine, Ministry of Health, Riyadh, Saudi Arabia.

Received date: July 07, 2021: Accepted date: August 24 2021: Published date: January 05, 2022

Citation: Sami Ayed Alshammary, Yahya Assiri, Reema AlRasheed, Yacoub Abuzied, Igbal Abelati Mahgoub and Khalid Alqumizi (2022) Satisfaction and Experience of Palliative Patients with 24/7 Hotline Service During the COVID-19 Pandemic in Saudi Arabia. J. Clin Case Rep and Stu 3(2); DOI: 10.31579/2690-8808/085

Copyright: () 2022 Sami Ayed Alshammary. This is an open access article distributed under the Creative Commons Attribution License, which permits unrestricted use, distribution, and reproduction in any medium, provided the original work is properly cited.

\begin{abstract}
Background: The COVID-19 pandemic prompted a number of shifts on healthcare. Conventional face-to-face visits were shifted during lockdown to virtual ones. Palliative care (PC) virtual visits have had high satisfaction rates, especially with patients in remote areas. Due to a number of factors, further studies are needed to develop tools that can be helpful and cost effective in improving patient's quality of life.

Objective: Our aim is to learn the main reasons palliative patients in Saudi Arabia sought help via calling the free 24/7 hotline and to discuss the hotline's satisfaction and effectiveness in solving the palliative patient's concerns during COVID-19.

Methods: A cross-sectional sample analysis was obtained from 214 patients from different regions in Saudi Arabia. A total number of 843 calls were made to the 24/7 PC hotlines from the period of 17 April 2020 to 28 February 2021, shortly after COVID-19 pandemic began. The purpose of the call, the caller's relationship to the patient, the status of the complaint, and the satisfaction rate were collected at the end of the call through a voluntary phone survey.

Results: The primary reasons that palliative patients called the hotline were: $30 \%$ for medication refills, $(n=247), 24.7 \%$ for medical complaints, $(n=205), 15.8 \%$ were for booking a new appointment $(n=131)$. Patients themselves accounted for $27.8 \%$ of the callers and patient's sons/daughters accounted for $51.3 \% .85 \%$ of patients said that their issue had been resolved by the end of the call and $89 \%$ of our sample were happy with the service provided through the hotline.

Conclusion: The 24/7 hotline service for PC patients in Saudi Arabia was successful in its application and resulted in a high level of satisfaction among a wide sample of participants. The main reasons palliative patients reached out were to request medication refills, seek assistance with a medical complaint, and to book a new appointment. Our hotline service effectively solved $85 \%$ of patients' issues.
\end{abstract}

Keywords: palliative care, virtual care, telemedicine, patient satisfaction, COVID-19

\section{Introduction}

Telemedicine is a division of medicine that deals with patients via telecommunication networks to deliver treatment from a distance [1]. Telemedicine has been increasingly used worldwide despite earlier resistance from healthcare practitioners who felt that it would endanger 
healthcare integrity and clinical results [1]. COVID-19 pandemic, however, has considerably accelerated the existence of telemedicine and digital health [2]. Telemedicine became an inevitable choice when patients and their families were under lockdown and had to cancel their in-person appointments for multiple reasons [2]. In New York, an 8729\% increase in video visits was observed during the COVID-19 pandemic (compared to before in March 4, 2020), and video visits were associated with higher satisfaction rates [3].

The World Health Organization (WHO) describes Palliative Care (PC) as the branch of medicine that improves the quality of life of patients and their families who are experiencing life- threatening disease (4). It is achieved through prevention and relief of suffering by early diagnosis, thorough evaluation, and treatment of pain and other difficulties such as medical, psychosocial, and spiritual issues [4].

Telemedicine use in palliative care has long been accepted, but the literature on virtual palliative care-specific advantages and drawbacks is still scarce $[1,5]$. Many considerations play unique roles in the virtual treatment of PC patients, such as practical, clinical, and ethical factors [5].

The benefits of video consultations are summarized as being practical, inclusive of a multidisciplinary team, and that also promotes collaboration between healthcare practitioners [6]. However, empathic patientphysician partnership was criticized in simulated environments, partially because PC specialists did not feel secure addressing critical and emotional issues through a physical distance [6].

\section{Problem statement}

Saudi Arabia's unique cultural values and computer literacy are completely different from other countries and are making rapid progress, which is why it is important to investigate their desires for virtual treatment. Shortly after the pandemic, PC patients in Saudi Arabia were helpless and distraught because their access to healthcare became interrupted and their fear of catching COVID-19 was increasing.

A multi-factor analysis to assess the option of virtual visits related to PC was undertaken in the United Kingdom in 2020 [5]. Stakeholders preferred virtual treatment because it decreases utilization of Personal Protective Equipment (PPE), decreases uncertainty of the COVID-19 pandemic, and improves time regulation [5].

Families and patients preferred a virtual visit because of its convenience, privacy, and someeven preferred it because it allowed them to read lips, which was not an option under mask mandates in office environments [5]. Telehealth in palliative home care is thought to be accessible and could potentially improve the quality of specialized palliative home care [6]. According to one study, patients and health professionals in Specialized Palliative Care have been actively engaged with telemedicine in PC and have been able to cooperate and make decisions on their health [6].

Visits for evaluation of chemotherapy were shifted to a virtual setting in Texas, USA during COVID-19 [7]. Although 73\% reported higher satisfaction, $65 \%$ of patients said they would prefer in person visits, suggesting that there is a distinction between virtual palliative care and other disciplines in telemedicine [7]. Another study discussing telemedicine's role in long-term care of radiation oncology found teleoncology to be more efficient and resulted in the reduction of cost for patients, especially to those living in remote areas [8]. It also found that tele-oncology allowed for continuity of care although its benefits are more evident in lower risk patients and is not a substitute for a medical visit, but more of a supplementary tool [8]. However, 50\% of oncologist deemed virtual prescription of chemotherapy not preferable [7]. Most oncologists, over $80 \%$, preferred hormonal and medical therapy virtual prescriptions [7].

Some argue that telephone consultations require a wealth of experience and skills, because it might miss important cues that can affect patients negatively [8]. Telephone encounters compared with video visits were found to be narrower in focus and tend to suppress the patient's ability to express his concerns naturally [11]. A study analyzed factors associated with favorability of telemedicine among oncology patients and found that the only factor that had a statistically significant relationship with telemedicine satisfaction was higher education level of the patient [14].

In a large tertiary hospital in Riyadh, Saudi Arabia, out of 200 patients, 84 were seen in virtual clinics over a period of four months, from March to May 2020 (4). These and multiple national virtual clinics were set up by the Ministry of Health (MOH) to respond to critical problems and offer treatment options to palliative care patients in a timely manner [4]. A hotline service was established by $\mathrm{MOH}$ to connect patients to these clinics and ensure continuity of care aiming to enhance PC patient's quality of life. A brief survey followed the call aiming to measure PC patient's satisfaction of this service.

\section{Materials and Methods Study design and setting}

This cross-sectional study was conducted in Saudi Arabia. As per the 2019 statistics by the Ministry of Municipal and Rural Affairs, the population in these regions is estimated at 2,595,762 people, constituting around $7.6 \%$ of Saudi Arabia's population [18]. The population has been growing at a rate of $1.5 \%$ in the last decade [18].

This is a cross-section analysis in which 214 patients evaluated their experience upon concluding a call to the regional hotline set up for palliative patients Saudi Arabia during the COVID-19 pandemic. A hotline was set up by the Palliative Care team at the Ministry of Health to address palliative patients concerns in these regions. This research was performed by analyzing the results of a brief telephone survey that followed the call to the palliative hotline service. Calls were received by three qualified health professionals, a clinical pharmacist with expertise in palliative pharmacy preparations, and two nurses with two or more years of experience in palliative care. The call receivers had full access to patients' electronic records and were able to view recommendations, specific issues, current medications, and care plan. A list of guidelines and a step-by-step approach was provided to them. In addition, they were able to access resources to guide their patients.

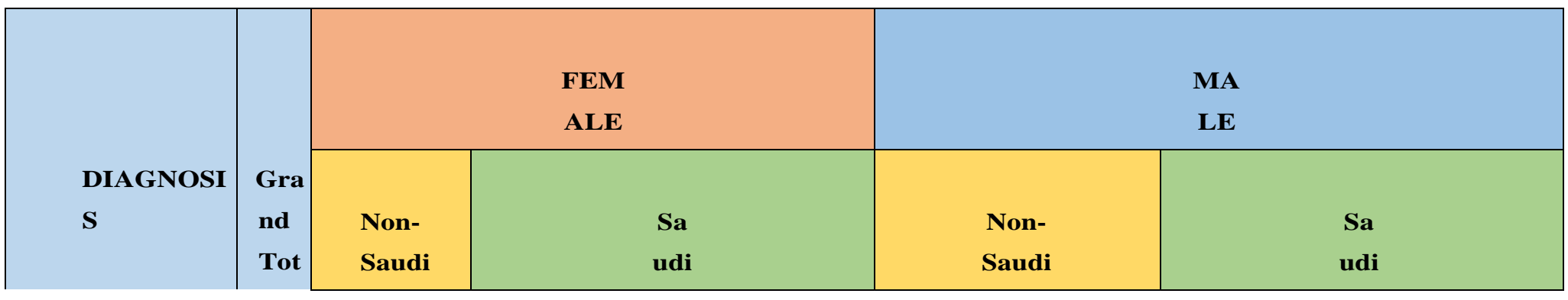




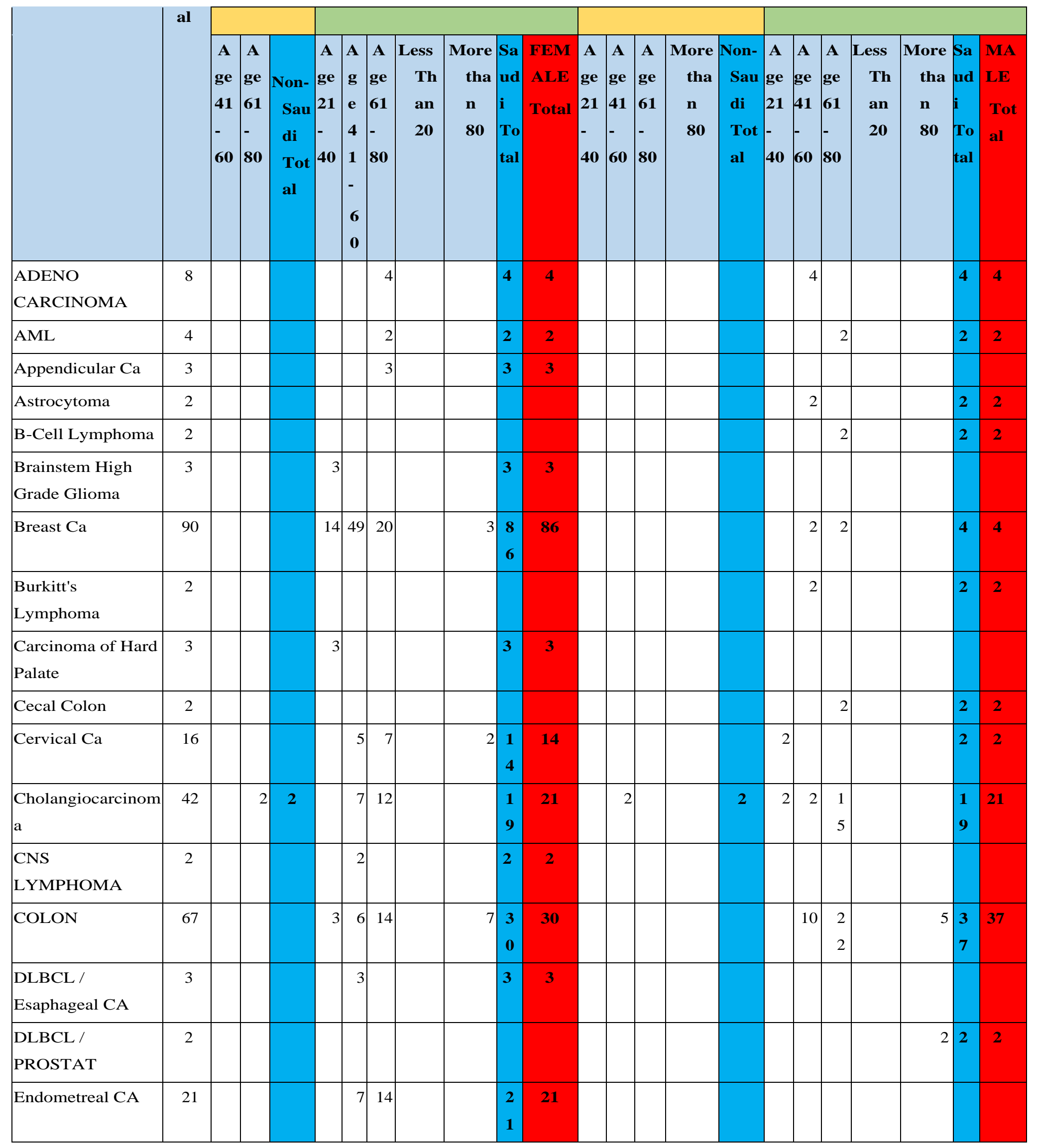




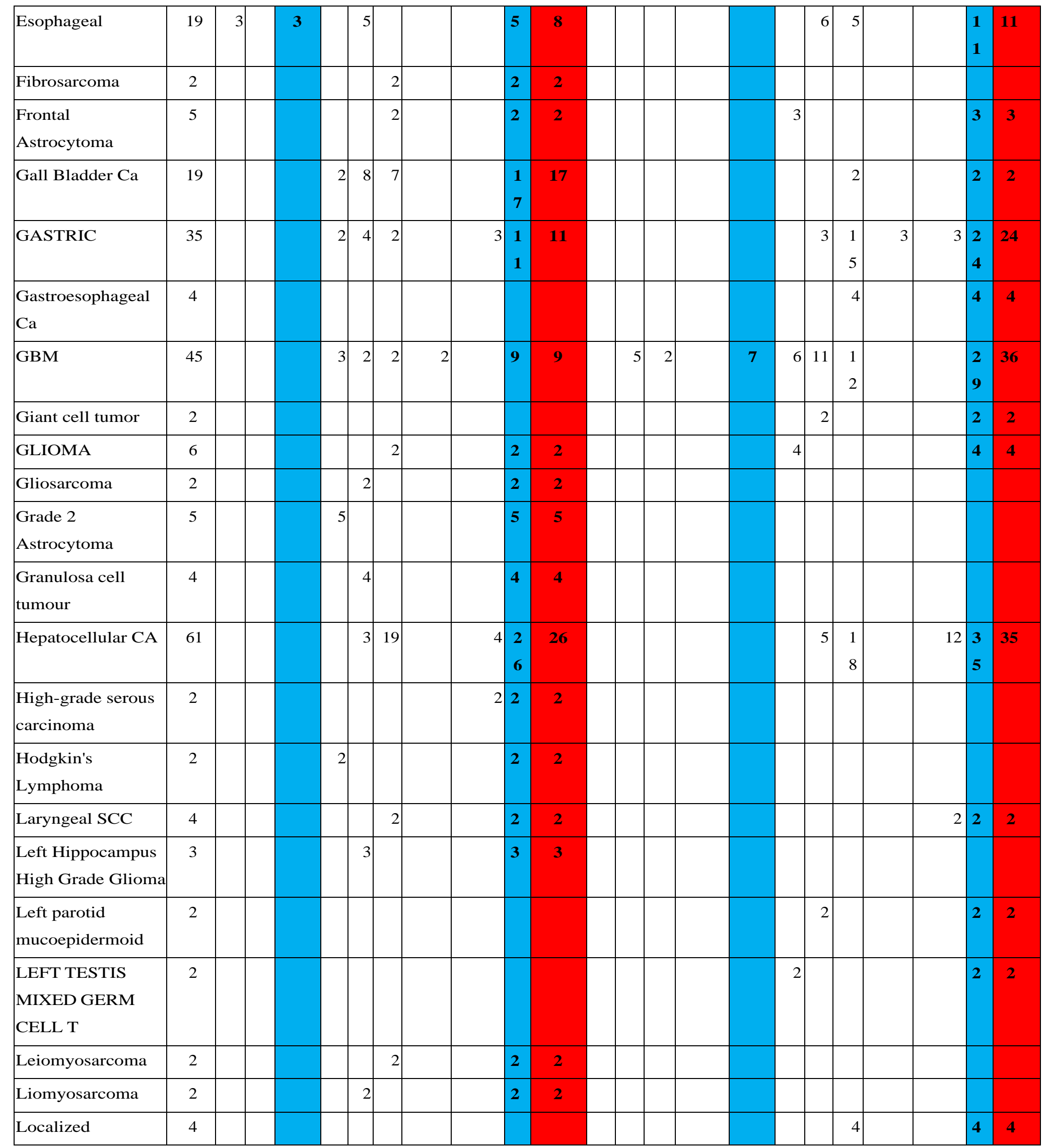




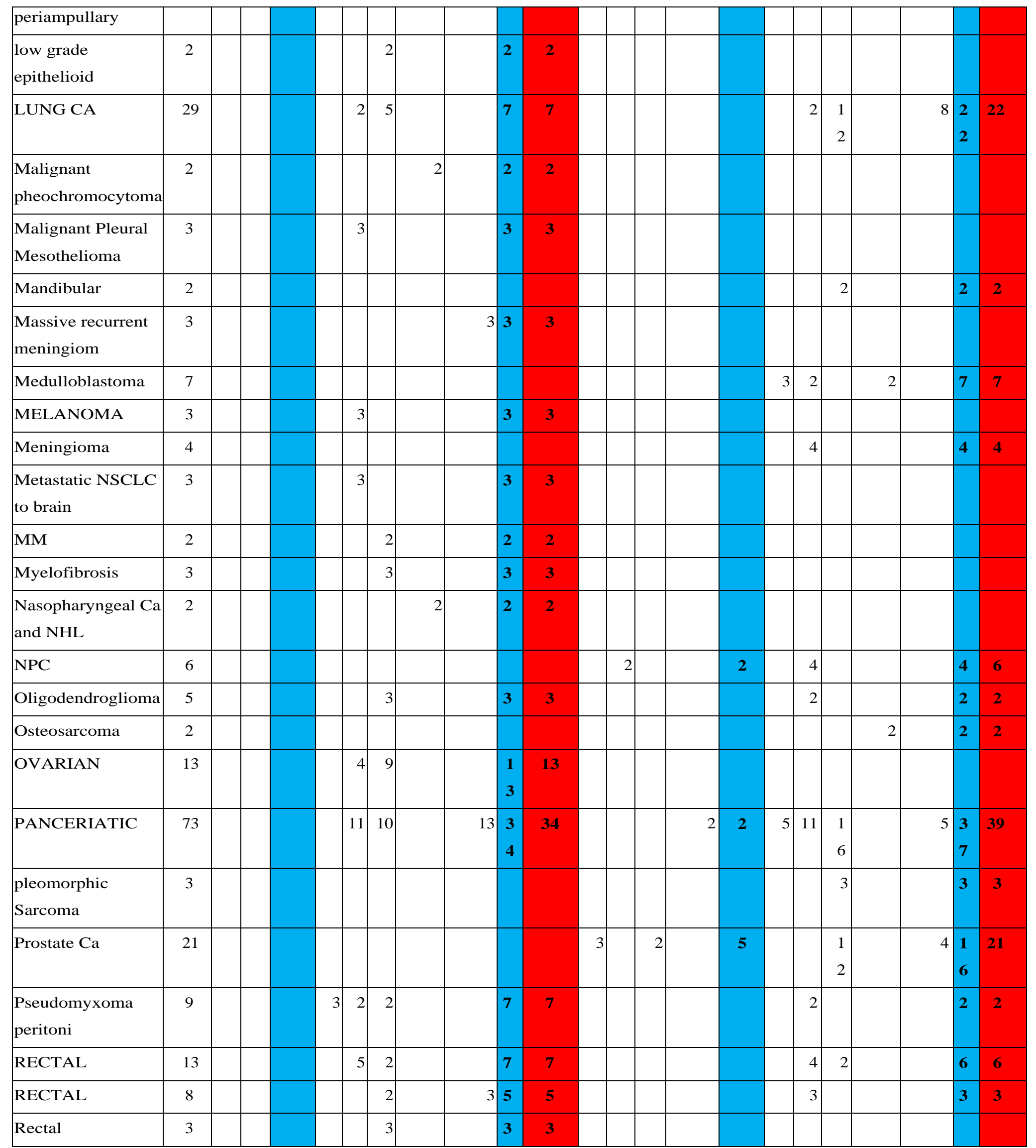




\begin{tabular}{|c|c|c|c|c|c|c|c|c|c|c|c|c|c|c|c|c|c|c|c|c|c|c|c|}
\hline \multicolumn{24}{|l|}{ Periampillary Ca } \\
\hline Recto Sigmoid Ca & 2 & & & & & & 2 & & & 2 & 2 & & & & & & & & & & & & \\
\hline RECTOSIGMOID & 7 & & & & & 2 & & & & 2 & 2 & & & & & & & & 5 & & & 5 & 5 \\
\hline Rectosigmoid & 3 & & & & & 3 & & & & 3 & 3 & & & & & & & & & & & & \\
\hline Rectum Ca & 5 & & & & & & & & & & & & & & & & 2 & & 3 & & & 5 & 5 \\
\hline $\begin{array}{l}\text { Recurrent Astro } \\
\text { Sytoma }\end{array}$ & 2 & & & & & 2 & & & & 2 & 2 & & & & & & & & & & & & \\
\hline Renal Cell Ca & 16 & & & & & 4 & 2 & & & 6 & 6 & & & & & & & 4 & & & & $\mathbf{1}$ & 10 \\
\hline $\begin{array}{l}\text { Right check } \\
\text { invasive SCC }\end{array}$ & 4 & & & & & & & & & 4 & 4 & & & & & & & & & & & & \\
\hline $\begin{array}{l}\text { Rt mandibular } \\
\text { Adenoid cystic ca }\end{array}$ & 3 & & & & & & & & & & & & & & & & & & 3 & & & 3 & 3 \\
\hline SARCOMA & 5 & & & & & 2 & & & & 2 & 2 & & & & & & & & 3 & & & 3 & 3 \\
\hline $\mathrm{SCC}$ & 8 & & & & & 2 & & & & 2 & 2 & & & & & & & 2 & 2 & & 2 & 6 & 6 \\
\hline Sigmoid Ca & 2 & & & & & & & & 2 & 2 & 2 & & & & & & & & & & & & \\
\hline $\mathrm{SSC}$ & 2 & & & & & & & & & & & & & & & & & 2 & & & & 2 & 2 \\
\hline SSC Anal & 2 & & & & & & & & & & & & & & & & & 2 & & & & 2 & 2 \\
\hline $\begin{array}{l}\text { Submandibular } \\
\text { SCC }\end{array}$ & 3 & & & & & 3 & & & & 3 & 3 & & & & & & & & & & & & \\
\hline TCC bladder & 3 & & & & & & & & & & & & & & & & & & & & & 3 & 3 \\
\hline THYROID & 14 & & & & & & 4 & & & 6 & 6 & & & & & & 3 & 2 & & & & 8 & 8 \\
\hline $\begin{array}{l}\text { Thyroid / Cervix } \\
\mathrm{Ca}\end{array}$ & 2 & & & & & & & & 2 & 2 & 2 & & & & & & & & & & & & \\
\hline Tongue carcinoma. & 3 & & & & & & & & & & & & & & & & & 3 & & & & 3 & 3 \\
\hline $\begin{array}{l}\text { UNKNOWN } \\
\text { PRIMARY }\end{array}$ & 11 & & & & & & 5 & & & 5 & 5 & & & & & & 3 & & 3 & & & 6 & 6 \\
\hline Urinary Bladder $\mathrm{Ca}$ & 3 & & & & & 3 & & & & 3 & 3 & & & & & & & & & & & & \\
\hline Uterine Ca & 14 & 2 & & 2 & & 5 & 4 & & & 9 & 11 & & & & & & & & 3 & & & 3 & 3 \\
\hline $\begin{array}{l}\text { Uterine } \\
\mathrm{Ca} / \text { Cervical }\end{array}$ & 4 & & & & & 4 & & & & 4 & 4 & & & & & & & & & & & & \\
\hline $\begin{array}{c}\text { Grand } \\
\text { Total }\end{array}$ & 843 & 5 & 2 & 7 & 40 & $\begin{array}{r}17 \\
5\end{array}$ & $\begin{array}{r}17 \\
6\end{array}$ & 6 & $\begin{array}{l}5 \\
0\end{array}$ & $\begin{array}{r}44 \\
7\end{array}$ & 454 & 3 & 9 & 4 & 2 & 18 & 35 & $\begin{array}{r}10 \\
0\end{array}$ & $\begin{array}{c}17 \\
4\end{array}$ & 7 & $\begin{array}{l}5 \\
5\end{array}$ & $\begin{array}{l}3 \\
7\end{array}$ & 389 \\
\hline
\end{tabular}




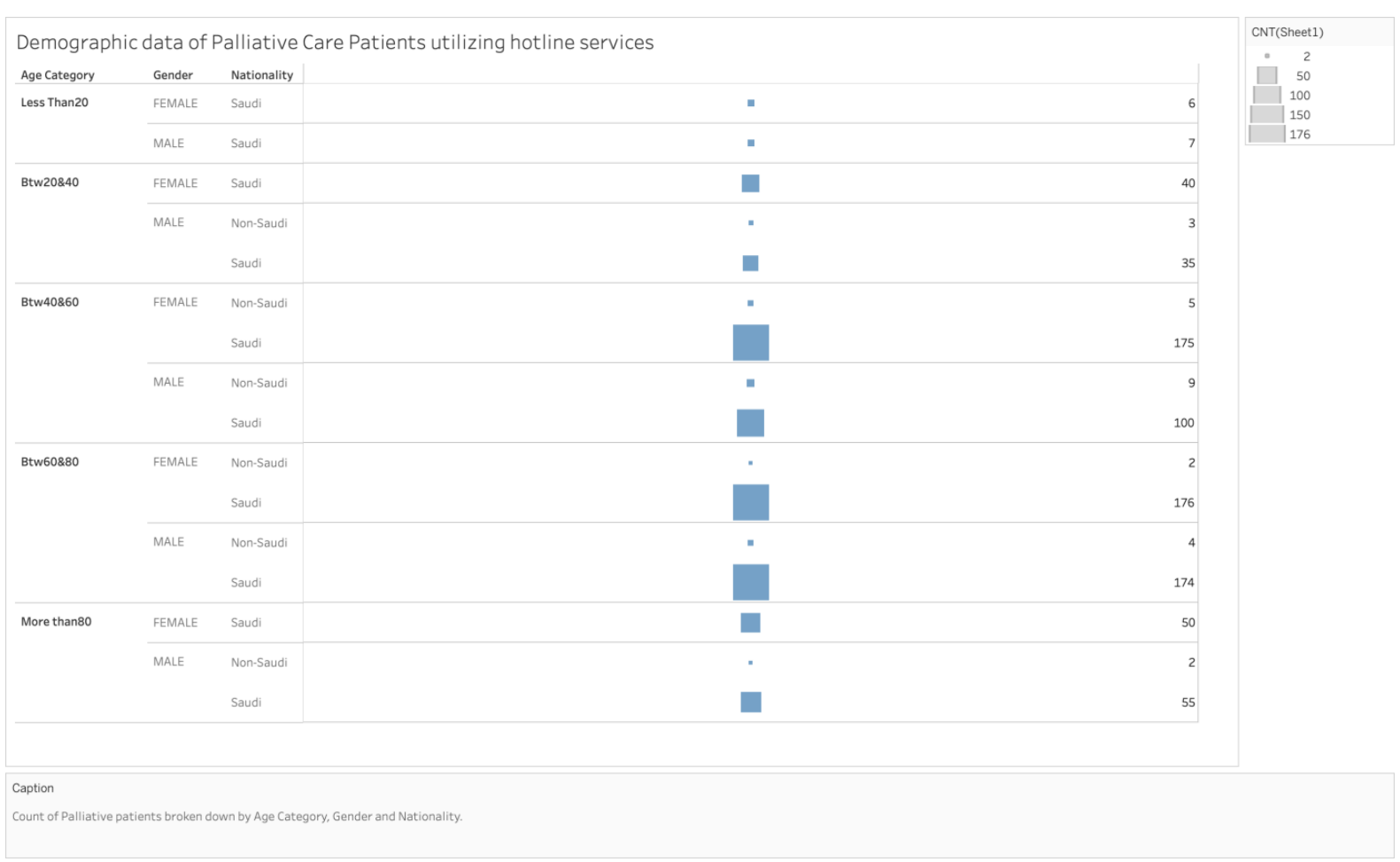

\section{Strategy}

A validated voluntary telephone poll consisting of five questions aimed to generate patient's opinion about the hotline instituted by $\mathrm{MOH}$ to enhance the patient's virtual palliative care experience. The survey was validated by 2 independent doctors in $\mathrm{MOH}$, a palliative care consultant, and a family medicine consultant. Data was entered on a separate Excel sheet and transferred to SPSS version 25.0 for analysis. All palliative patients who called the hotline from 17 April 2020 to 28 February 2021 with a PC diagnosis were included, and those who did not have a PC diagnosis were excluded.

\section{Results}

214 patients, $51 \%$ of which are females, have called the hotline 843 times "between" 17 April 2020 to 28 February 2021. In general, patient satisfaction in regards to their experience was exceedingly high, as $90 \%$ of callers reported satisfaction by the end of the call.

The main reasons for the call to the hotline are summarized in Figure 1. Our results show that $30 \%$ of palliative patient's reached out to the hotline for medication refills $(n=254), 24.7 \%$ called for medical complaints $(n=205)$, and $15 \%$ called to book a new appointment $(n=131)$. However, $29.8 \%$ of the calls were for other unspecified reasons $(n=248)$. Interestingly, patients themselves were the inquiring caller in only $27.8 \%$ of the cases while almost half of the callers, $47 \%$, were the patient's son or daughter $(n=433)$. Figure 2 shows the caller's relationship to the patient.

$86.7 \%$ of callers stated that their problem was solved by the call $(n=731)$, and $10.8 \%(n=91)$ of callers said their issue was partially solved by the phone call, and only $2.5 \%(n=21)$ said their problem was not solved.

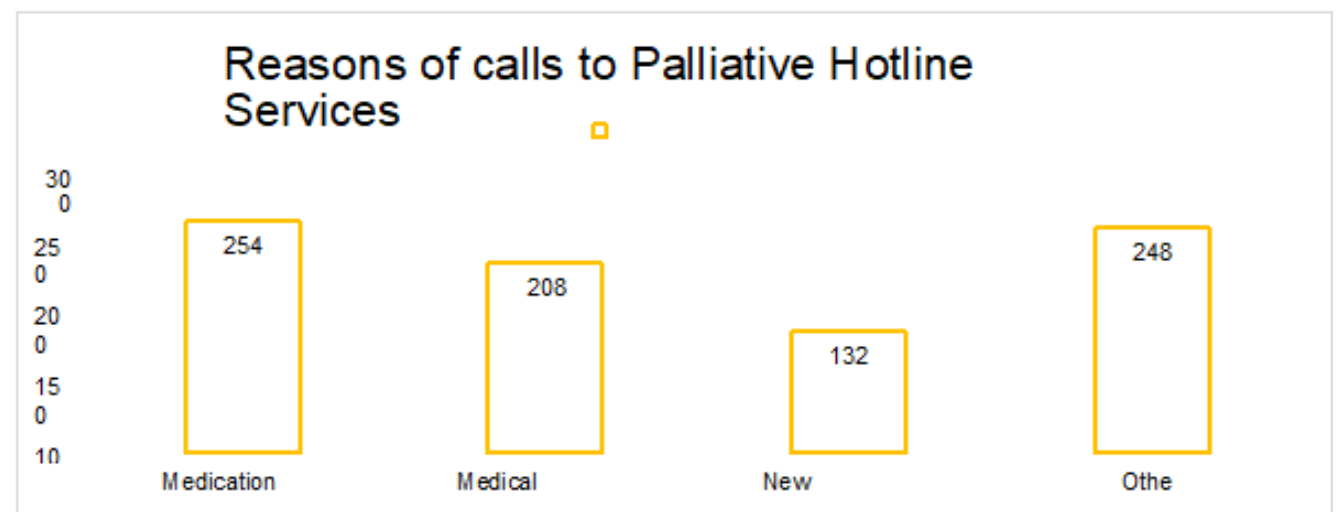

Figure 1 Main reasons patients called the palliative hotline 


\section{Who made the call for the}

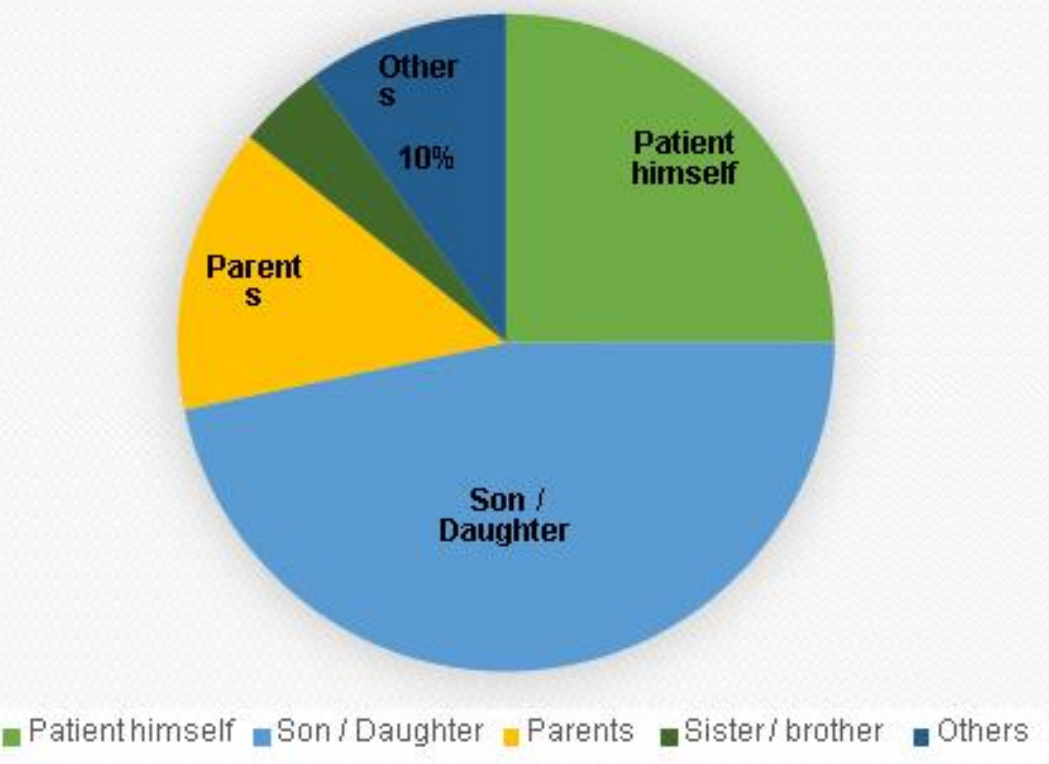

Figure 2 Caller's relationship to the patient

\section{Discussion}

Our experience in setting up a free hotline call center for palliative patients in Saudi Arabia was very successful in connecting patients to their providers and solving both logistical and medication supply issues. During COVID-19 pandemic, and particularly in its early phases, Saudi Arabia enforced strict guidelines and stay at home orders in several provinces to combat COVID-19 spread. Travel between cities and major regions was highly discouraged except for certain essential workers. Most patients were discouraged from coming to the hospital, specifically palliative patients were encouraged to stay at home because coming to the hospital would mean that they will mostly be unaccompanied by their caregivers, which is a sensitive and an integral part of palliative patients' social support. The $24 / 7$ hotline for palliative patient was a new initiative taken to help adapt to the stress caused by the interruption of patient's visits during COVID-19 pandemic. Patients in Saudi Arabia frequently travel more distances to access care, hence this new measure can be very helpful to enhance their access to healthcare. The hotline created a costeffective bridge for palliative patients in both rural and urban regions to reach their providers without needing a virtual appointment nor an inperson visit. The number of calls received averaged 70 calls/ month, which indicate the need for similar resources that operate like an intermediate link between palliative patients and palliative care physicians without overwhelming the system. Prescriptions refills have been a pressing challenge for palliative care patients living far from hospitals who have been undergoing treatment for a long time. This has been further exacerbated by the COVID-19 pandemic. New resources are required to improve patient access and continuity of treatment for patients dealing with pain and end of life. Studies suggest that telemedicine utilization is effective in palliative care, and our study affirms that meeting patients' needs through virtual settings was satisfactory. Raffenaud, Amanda et al (2019) found that from the treating oncologist's perspective, tele-oncology was not superior to in-person visit for new patients, before chemotherapy sessions, and for high recurrence-risk cancer patients who require a physical examination.

Drug and prescription-related inquiries caused our patients discomfort, and care should be taken to ensure continuity of care through telemedicine and tele-pharmacy for palliative patients in times of crises. Telecommunication to manage inquiries and provide answers by experienced personnel has shown to be very beneficial for palliative patients in different regions. At the same time, virtual PC does not include a physical drive, greatly serving patients in remote areas. Based on our findings, we are advocating on behalf of PC patients to have legislative frameworks to ensure the healthcare system's coherence in terms of medication refills. A coherent and supportive system can further support palliative patient's rights to uninterrupted pain treatment during periods of large-scale changes.

Phone calls instead of video calls results in a particular form of conversation, and the investigation of the difference between these from a cultural perspective may provide useful insights. By looking into the gender distribution of the callers in our sample, we see that half of these callers were females and Saudi culture and religion is unique in that it necessitates female covering their hair, body, and face in many cases. These nuances can affect their preference of phone versus video visits. Telephone calls are cheap and easy to institute and use. They do not require network utilization and require fewer configurations compared to video visits. Also in Saudi Arabia, it is very common for caregivers, specifically daughters and sons, to assume responsibility of patients' information and healthcare decisions. As our study shows, almost $70 \%$ of the calls were made on behalf of the patient.

Confidentiality issues can arise when the patient is not the main caller, but in Saudi Arabia, it is common for daughters and sons to take over and this is often overlooked but should be carefully examined from an ethical perspective. Verification of the caller and ensuring granted access of the caller should be emphasized in phone calls more than videos, as it can be challenging to differentiate who the caller is. These instances should also be documented to enhance privacy and to avoid disclosing personal health information to unauthorized people.

Our findings are in line with the published literature; a palliative care hotline benefits patient considerably, has high satisfaction rates, and is 
particularly important in times of decreased access to healthcare.

Alshammaray S, et al. reported the results of patient satisfaction of virtual visits in palliative care at a tertiary hospital in Riyadh, the capital city of Saudi Arabia. $87 \%$ of patients seen in the virtual clinic rated the visit 5/5 [15]. In Vancouver, British Columbia, Canada, a palliative care telephone consultation evaluation study by Ridley JZ, Gallagher R. (2008) showed that most calls came from rural areas and the primary reason for calls was pain management, followed by gastrointestinal symptoms [19]. In Taiwan, Lin, M. H., Chen, H. N., \& Chen, T. J. (2020), piloted a personalized discharge end-of-life care to reduce frequency of dialing 24hour hotlines that resulted in 5-10\% decrease on the hotline, indicating significant need of patients near death to have access to phone educational and consultation hotlines [20].

The limitation of our study includes lack of direct availability of palliative care physicians in case an urgent inquiry beyond the scope of the call receiver. An integrated approach that links physicians and medical students to further expand the services and respond better to palliative care patients can be beneficial and can also result in decreased costs for the patients and physicians.

Based on Claire K. Ankuda, et al (2020) similar experience of setting up a crisis hotline run by medical students in Mount Sinai, New York to serve palliative patients during the pandemic, several principles and teachings were proposed [21]. The recommendations included staffing two full-time equivalent (FTE) palliative care physicians, in addition to four FTE physicians when call volumes are higher than usual [21].

Qualitative interviews are imperative to uncover desires and satisfaction in a broader manner and elicit needs and opinions of palliative patients and physicians overseeing their cases. Geospatial data was also not collected and could have been helpful to accurately measure cost effectiveness of instituting a more advanced model of hotline services to palliative patients in rural / remote areas. Other causes for calls, which amounted to $29.8 \%$, were also not elucidated and should have been properly investigated by call recipients. This research will act as a starting point for learning about the needs and perspectives of Saudi palliative patients when it comes to virtual palliative care.

Since the adoption of telemedicine was swift and inevitable, opinions and patient satisfaction must be elicited. This can provide a better framework to accessing care in future and present challenges and ensure continuity of care and quality of life for patients living in rural / remote areas. Therefore, palliative care helpline will probably stay and expand to longterm care and many other components of palliative care that aims to enhance patients' quality of life.

\section{Conclusion}

The experience of a telephone hotline for palliative care patients was regarded as a highly useful service and was also highly accepted by patients. It was cost effective and used very frequently by patients. Medication refills were the most common cause of repeated phone calls and efforts should be made to tend to the impact of the interruption of inperson care on medication availability for palliative care patients. Hotlines can serve as an effective bridge to care and decrease pressure on specialists by solving issues not requiring a visit to a physician but a visit to an institution.

\section{Ethical considerations}

\section{Ethics and consent to participate}

Participants were told of the intent of the research and of their right to reject participation. Ethical behavior has been preserved throughout data acquisition and during the analysis period. Participation in the study was voluntary and each subject had the right to withdraw from the study at any time without any repercussions. The institutional review board at King Fahad Medical City, Riyadh, Saudi Arabia gave ethical approval for this study. We used primary data collected by the research team. Consent for collecting data was granted by the chairman of the Palliative Care department.

\section{Declarations}

\section{Consent to Publish: N/A}

Availability of data and materials: All data generated and/or analyzed during this study included in this published article. The data are available from the corresponding author upon request.

Competing interests: The authors declare that they have no competing interests.

Funding: This research received no specific grant from public, commercial or non-profit funding agencies.

Authors' Contributions: Each person mentioned in this manuscript contributed significantly and in compliance with the standards of the International Committee of Medical Journal Editors.

\section{Acknowledgments}

We want to acknowledge North Business Unit (NBU), Health Holding Company, Riyadh, Saudi Arabia for technical support.

\section{References}

1. Funderskov, K. F., Boe Danbjørg, D., Jess, M., Munk, L., Olsen Zwisler, A. D., \& Dieperink, K. B. (2019) Telemedicine in specialised palliative care: Healthcare professionals' and their perspectives on video consultations-A qualitative study. Journal of clinical nursing, 28(21-22), 3966-3976.

2. Lally, K., Kematick, B. S., Gorman, D., \& Tulsky, J. (2021). Rapid Conversion of a Palliative Care Outpatient Clinic to Telehealth. JCO oncology practice, 17(1), e62-e67.

3. Ramaswamy A, Yu M, Drangsholt S, Ng E,Culligan PJ, Schlegel PN, Hu JC. (2020) Patient Satisfaction With Telemedicine During the COVID-19 Pandemic: Retrospective Cohort Study. J Med Internet Res, 22(9):e20786.

4. AlShehery, M. Z., Duraisamy, B., Zaidi, A., AlShehry, N. F., Zaidi, F. Z., et al. (2020). COVID-19 and Palliative Care Services: Comparative Patterns of Inpatient, Outpatient, and Consultation Services in a Tertiary Care Center in Riyadh. Cureus, 12(12), e11996.

5. Hawkins JP, Gannon C, Palfrey J. (2020) Virtual visits in palliative care: about time or against the grain? BMJ Supportive \& Palliative Care 10:331-336.

6. Steindal SA, Nes AAG, Godskesen TE, Dihle A, Lind S, Winger A, Klarare A, (2020) Patients' Experiences of Telehealth in Palliative Home Care: Scoping Review, JMed Internet Res 22(5):e16218.

7. Ajithraj Sathiyaraj, Hannah Lopez, Rakesh Surapaneni. (2021) Patient satisfaction with telemedicine for prechemotherapy evaluation during the COVID-19 pandemic. Future Oncology 17:13, 1593-1600.

8. Lewis, G., Hatch, S., Wiederhold, L., \& Swanson, T. (2020). Long-Term Institutional Experience With Telemedicine Services for Radiation Oncology: A Potential Model for LongTerm Utilization. Advances In Radiation Oncology, 5(4), 780782.

9. Chua, I. S., Jackson, V., \& Kamdar, M. (2020). Webside Manner during the COVID-19 Pandemic: Maintaining Human Connection during Virtual Visits. Journal of palliative medicine, 23(11), 1507-1509. 
10. Groot, M., Vernooij-Dassen, M., Crul, B., \& Grol, R. (2005). General practitioners (GPs) and palliative care: perceived tasks and barriers in daily practice. Palliative Medicine, 19(2), 111118.

11. Greenhalgh T, Vijayaraghavan S, Wherton J, et al. (2016) Virtual online consultations: advantages and limitations (VOCAL) study BMJ Open. 6: e009388.

12. Shirke, M. M., Shaikh, S. A., \& Harky, A. (2020). Implications of Telemedicine in Oncology during the COVID-19 Pandemic. Acta bio-medica: Atenei Parmensis, 91(3), e2020022.

13. Tashkandi E, Zeeneldin A, AlAbdulwahab A, Elemam O, Elsamany S, Jastaniah W, et al. Virtual Management of Cancer Patients in the Era of COVID-19 Pandemic. J Med Internet Res.

14. Raffenaud, Amanda et al. (2019) 'Utilizing Telemedicine in Oncology Settings: Patient Favourability Rates and Perceptions of Use Analysis Using Chi-Square and Neural Networks'. 115 $-127$.

15. Alshammaray S, Duraisamy B, Albalawi Y, et al. (March 26, 2019) Development of Palliative and End of Life Care: The Current Situation in Saudi Arabia. Cureus 11(3): e4319.
16. In L. De Lima, K. Pettus, J. Downing, S, Connor, J. Marston Palliative Care and Covid-19 Series - Briefing Notes Compilation (pp. 5-11) Houston: IAHPC Press.

17. Groot, M., Derksen, E., Crul, B., Grol, R., \& Vernooij-Dassen, M. (2007). Living on borrowed time: Experiences in palliative care. Patient Education And Counseling, 65(3), 381-386.

18. Saudi Cities Report 2019, Ministry of Municipal and Rural Affaris- UN Habitat report

19. Ridley, J. Z., \& Gallagher, R. (2008). Palliative care telephone consultation: who calls and what do they need to know? Journal of palliative medicine, 11(7), 1009-1014.

20. Lin, M. H., Chen, H. N., \& Chen, T. J. (2020). Changes in 24Hour Palliative Care Telephone Advice Service after the Introduction of Discharged End-of-Life Patients' Care Plans. International journal of environmental research and public health, 17(16), 5876.

21. Ankuda, C., Woodrell, C., Meier, D., Morrison, S., \& Chai, E. (2020). A Beacon for Dark Times: Palliative Care Support During the Coronavirus Pandemic. NEJM Catalyst, May 12, 2020.
This work is licensed under Creative Commons Attribution 4.0 License

\section{To Submit Your Article Click Here: Submit Manuscript}

DOI: $10.31579 / 2690-8808 / 085$
Ready to submit your research? Choose Auctores and benefit from:

* fast, convenient online submission

* rigorous peer review by experienced research in your field

* rapid publication on acceptance

* authors retain copyrights

* unique DOI for all articles

* immediate, unrestricted online access

At Auctores, research is always in progress.

Learn more auctoresonline.org/journals/journal-of-clinical-case-reportsand-studies 